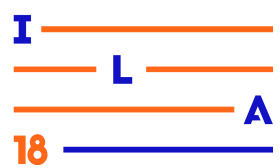

\title{
APLICACIONES GOOGLE Y ADULTOS MAYORES: UN TESTEO DE USABILIDAD SOBRE GDOCS Y GDRIVE
}

\author{
GOOGLE APPLICATIONS AND ELDERLY: GDOCS AND GDRIVE \\ USABILITY TEST
}

\author{
Javier Diaz ${ }^{1}$, Prof. \\ Sandra Baldassarri² ${ }^{2}$ D.Sc. \\ Ivana Harari ${ }^{3}$, M.Sc.
}

(1) Facultad de Informática- Universidad Nacional de La Plata e-mail: jdiaz@info.unlp.edu.ar

(2) Departamento de Informática e Ingeniería en Sistemas- Universidad de Zaragoza e-mail: sandra@unizar.es

(3) Facultad de Informática- Universidad Nacional de La Plata e-mail: iharari@info.unlp.edu.ar

Adultos mayores, Usabilidad, Aplicaciones web

Este artículo describe un testeo de usabilidad sobre GDocs y GDrive, realizado a más de cien personas entre 68 y 90 años de edad. Se incluyen detalles sobre la muestra de usuarios, metodología y análisis de resultados que permitirán determinar cuan usables son estas importantes aplicaciones considerando esta comunidad especifica.

\section{Elderly, Usability, Web applications}

This paper describes a GDocs and GDrive's usability test where more than one hundred of 68 to 90 years-old people participated. It includes details of the user profiles, methodology and result analysis that permits to know the degree of usability of these important applications considering this specific community.

\section{Introducción}

El ser humano desde miles de años han utilizado textos impresos como medio de expresión. Ahora, cuentan con aplicaciones de software que no sólo permiten la edición de textos, su revisión y borrado, sino que a su vez posibilitan hacerlo en forma remota, compartirlo y dejar que esas acciones puedan ser realizadas en conjunto con los demás.

Este escenario colaborativo y remoto de procesamiento de textos, es lo que brindó Google desde 2007, con la aplicación de ofimática GDocs y desde abril de 2012, con GDrive, el servicio en la nube de almacenamiento de archivos (MANSOR, 2012).

Debido a la popularidad y predominio de estas aplicaciones, las mismas definen un estándar de uso tanto de la administración de archivos como de las aplicaciones de oficina en la nube. Por tal motivo se hace imprescindible analizarlas en forma integral desde la perspectiva de la interacción hombre máquina HCI, de la usabilidad y experiencia de uso. Más aún se hace ineludible trabajar con comunidades vulnerables como desde el perfil de un adulto mayor, donde se requiere maximizar las cualidades de un producto usable y accesible, en pos de brindar mayor soporte, simpleza y asistencia en la interacción (FINN et al., 2014).

Los adultos mayores, con un crecimiento demográfico cada vez mayor debido al aumento de la esperanza de vida, van a convertirse en los futuros usuarios de las aplicaciones de software. $\mathrm{Su}$ acercamiento a la tecnología constituye una alternativa para mejorar su calidad de vida. (CASAMAYOU et al., 2017).

En este sentido, el presente artículo analiza la experiencia de los usuarios de edad avanzada frente a las aplicaciones de GDocs y GDrive, mediante el desarrollo de un testeo de usabilidad a 120 personas entre 68 y 90 años de edad. Se describe la muestra de los participantes, las estrategias utilizadas como los resultados obtenidos. 


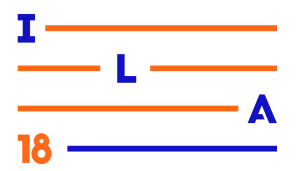

Esto permite fundamentalmente determinar si estas aplicaciones están preparadas para ser usadas por una comunidad de personas que no nació con las nuevas tecnologías, para saber si los adultos mayores pueden utilizarlas con facilidad, aprovechando sus aspectos de compartición, colaboración y de distancia, ya que es en la lejanía el lugar donde los recursos residen y se encuentran disponibles (NIA y NLM, 2002).

\section{El testeo de usabilidad}

El término usabilidad es un anglicismo que significa facilidad de uso. Su definición formal se refiere al grado de eficacia, eficiencia y satisfacción con la que usuarios específicos pueden lograr objetivos específicos, en sus contextos particulares de uso (ISO, 2018).

El concepto de usabilidad tiene muchas acepciones y puede ser analizado desde diferentes perspectivas, desde lo empírico y cuantitativo, desde lo normativo y de diseño, como hasta desde lo subjetivo y cualitativo. No sólo puede ser definido como atributo de calidad de un producto, sino como un proceso, metodología de diseño y evaluación. (GREMILLION et al., 2012).

Uno de los métodos formales para determinar si un sistema es usable consiste en observar usuarios interactuando con el mismo sistema, registrando cómo se manifiestan, cómo se desempeñan, qué es lo que sienten y cuáles son las estrategias que emplean para llevar a cabo actividades reales sobre el producto a evaluar (NIELSEN et al., 2004).

Los testeos de usabilidad son fundamentales para esto. Se observan las sesiones recabando información de interés sobre la interacción, tales como problemas encontrados, consultas que realizan, interrupciones, comentarios, y se registran los resultados para un análisis posterior. Los datos se recaban mediante la utilización de planillas y la técnica de observación directa (HASSAN et al., 2009).

Lo que se plantea aquí es medir la usabilidad en términos de performance y satisfacción, donde se considera circunstancias específicas de uso. En las siguientes secciones, se va a describir la muestra de participantes, las estrategias empleadas, detalles del testeo, como también los resultados obtenidos.

\subsection{Objetivo del testeo de usabilidad}

El objetivo del testeo de usabilidad es determinar usables son los servicios de edición, compartición, coautoría y almacenamiento remoto provisto por GDocs y GDrive por parte de la gente mayor. Las actividades que se plantearán implicarán el uso de ambas aplicaciones como si fuera un todo, ya que dentro de las intenciones del usuario será tanto la edición de documentos como su almacenamiento.

\subsection{Planificación del testeo de usabilidad}

Desde varios años, la Universidad Nacional de La Plata de Argentina, tiene un convenio con PAMI para la realización de cursos de capacitación en distintas disciplinas destinados a adultos mayores (DIAZ, 2012). En este marco se realizó la convocatoria para participar en el proceso de evaluación, que se llevó a cabo durante los periodos lectivos 2016 y 2017.

En total, hubo 120 personas interesadas, a las que se las agruparon en comisiones de 25 a 30 para que pudieran utilizar una PC por persona en el momento del test, utilizando las máquinas disponibles en la sala de computadoras de la Facultad de Informática ${ }^{1}$.

Para cada comisión se planificaron las siguientes actividades:

- Entrevistas, para considerar el perfil de los participantes, sus conocimientos sintácticos y semánticos respecto al uso de la computadora, al procesamiento de textos, sobre aplicaciones web colaborativas, acceso a la nube, entre otros.

- Charlas introductorias, se organizaron de dos a tres encuentros para explicar las aplicaciones a evaluar como también detalles del proceso de evaluación.

- Desarrollo del testeo de usabilidad, donde los adultos mayores concurrieron de dos a tres veces a realizar las actividades solicitadas, en jornadas de 3 horas.

- Charla final de reflexión, donde se los citó en una jornada de una hora, donde contaron sus experiencias en el proceso evaluativo.

1 Sala de PC equipada con 28 PCs de doble booteo Win 7 y Ubuntu Linux, con 1,35 GHz y 4GbRAM 


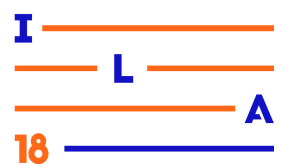

Con esta planificación, el proceso evaluativo por comisión tenía una duración entre dos a tres semanas. Una semana con la participación directa de los adultos mayores, y el resto para la preparación inicial como para el posterior análisis de los resultados por parte del equipo evaluador.

Es importante recalcar que ellos entendieron el sentido del proceso evaluativo que iban a impartir, las actividades que iban a realizar y la forma de trabajo. Ellos no iban a ser el foco de evaluación, sino lo que se iba a analizar era el software en sí.

Manifestaron tranquilidad al entender que los posibles costos, dificultades, errores que pudieran cometer en las pruebas de interacción con los productos podrían, no solo deberse a las limitaciones de la edad y a su falta de destrezas que ellos siempre argumentan, sino también a problemas propios de diseño y falencias de usabilidad que los mismos productos presentan y que era justamente, nuestro objeto de estudio y evaluación.

\subsection{Muestra de participantes}

La muestra de participantes con lo que se realizó el testeo de usabilidad de GDocs y GDrive fue de 120 personas entre 68 y 90 años, de la entidad nacional PAMI. Con ellos, se llevó a cabo una investigación de usuarios mediante entrevistas y cuestionarios para conocer sus conocimientos previos y experiencias (KRUG, 2006).

De acuerdo a esto, se puede decir que:

- La mayoría de la muestra eran mujeres, un $67,5 \%$

- Un 55\% tenían solo nivel de estudios primario, $26,66 \%$ secundario y un $12,50 \%$ terciario o universitario. El resto sin formación formal.

- El $88,33 \%$ es jubilado o pensionado, donde un $48 \%$ no tiene computadora en su hogar.

- Un 48,33\% nunca usó un procesador de textos y un $35 \%$ lo usó pocas veces.

- Un 32\% nunca oyó sobre Google, un $45 \%$ no entiende bien qué es y el resto lo asoció con un buscador, con el correo electrónico o mapas, pero solo una persona lo asoció con una suite de ofimática en la nube.

- Con respecto a las aplicaciones específicas de GDocs y GDrive solo 4 personas indicaron haber escuchado de ellas sin saber realmente qué son.

- La mayoría, un 64,10\%, querría aprender a utilizarlas para poder actualizar sus conocimientos, un $77,5 \%$ para entender y acercarse a las nuevas generaciones y establecer contacto con ellos y un $91,66 \%$ porque cree que con el uso de las TICs pueden mejorar sus habilidades cognitivas y motrices.

\subsection{Desarrollo del test de usabilidad}

El testeo de usabilidad consistió en el desarrollo de 10 actividades en GDocs y GDrive que representan intenciones habituales que podrían tener los usuarios en mente. Las mismas fueron llevadas a cabo en tres encuentros para que los alumnos tuvieran tiempo para realizarlas con tranquilidad.

Previo a la realización del testeo, se les crearon cuentas de Google a las personas que la requerían. Entre las 120 personas, un 30\% tenían cuenta creada de GMail, la mayoría de ellas habían sido creadas por terceros o por haber realizado algún curso previo.

Las actividades se listan en la Tabla 1.

\begin{tabular}{|l|l|}
\hline 1 & Ingresar a GDrive y crear un documento \\
\hline 2 & $\begin{array}{l}\text { Escribir una nota invitando a un evento y colocarle el nombre } \\
\text { "Invitación" al trabajo realizado. }\end{array}$ \\
\hline 3 & Insertar una imagen relativa al tema del evento. \\
\hline 4 & $\begin{array}{l}\text { Compartirlo a una persona con permiso de edición y a otra } \\
\text { solo para comentar. }\end{array}$ \\
\hline 5 & Crear una carpeta llamada Trabajos en GDrive \\
\hline 6 & Mover la nota de invitación realizada por Ud. a dicha carpeta. \\
\hline 7 & Descargar en el disco la nota realizada por Ud. \\
\hline 8 & $\begin{array}{l}\text { Buscar algún trabajo compartido por otro compañero suyo y } \\
\text { averiguar a quienes más se les compartió dicho trabajo. }\end{array}$ \\
\hline 9 & $\begin{array}{l}\text { Dejar un comentario a algún trabajo compartido que tenga } \\
\text { permiso para hacerlo. }\end{array}$ \\
\hline 10 & Salir de la aplicación cerrando correctamente la sesión. \\
\hline
\end{tabular}

Tabla 1: Listado de actividades

La planilla de registro de los resultados observados al realizar las actividades designadas por parte de la gente mayor, consistía en cuatro partes. En una primera parte, se registraba cuestiones de eficacia, si la actividad se había realizado en forma simple, compleja o directamente no se había logrado.

En una segunda parte, se explicaban cuestiones de eficiencia, es decir las dificultades encontradas ya sea porque realizó la actividad en forma compleja o porque directamente no pudo desarrollarla. Los motivos considerados fueron que se desorientó, no encontraba la forma de hacerlo o no lo entendía. 


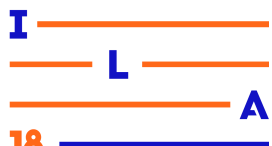

En la tercera parte, se registraron los tiempos de realización que también describen cuestiones de eficiencia y el grado de satisfacción que sentían los usuarios luego de realizar la actividad.

Los umbrales estimados para los tiempos de realización fueron consensuados previamente y analizados según pruebas que se llevaron a cabo con usuarios mayores. Estos se clasificaron en:

- Rápido: menos de 5 minutos de realización.

- Lento: entre 5 a 10 minutos.

- Muy lento: más de 10 minutos.

Con respecto al grado de satisfacción, se consideró:

- Alto: cuando el alumno manifiesta alegría y reconocimiento de la labor realizada.

- Medio: cuando está contento pero con algunas quejas sobre la experiencia.

- Bajo: cuando el alumno se queja mucho, se enoja o se siente frustrado.

La Tabla 2 muestra la planilla utilizada:

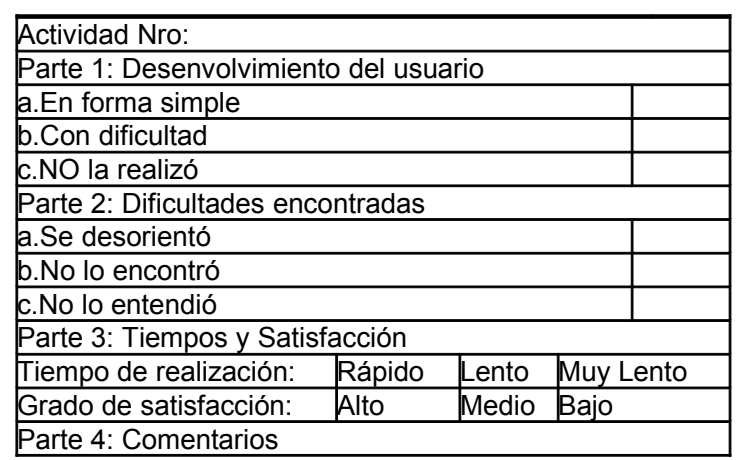

Tabla 2: Planilla de Registro

En la parte final de la planilla, se reservó espacio para los comentarios donde el observador anotaba algunas expresiones vertidas por el usuario.

Las planillas fueron completadas por los expertos en HCI con la técnica de observación directa. Los resultados arrojados se muestran en la Tabla 3, que se mostrarán en la sección siguiente. Detalles de los mismos se encuentran en la sección 3.

\subsection{Resultados obtenidos}

El proceso de evaluación de usabilidad de GDocs y GDrive por parte de usuarios de edad avanzada fue una experiencia enriquecedora. Fue notable el interés de los participantes en explicar qué sentían, en qué se equivocaban, los caminos andados, qué elementos de la interfaz los confundían. Muchos opinaban sobre soluciones posibles.

Los resultados provenientes de las partes 1 y 2 de la planilla, se pueden observar en la Tabla 3 . Los valores están expresados en porcentajes y en cantidad de personas.

\begin{tabular}{|c|c|c|c|c|c|c|}
\hline \multicolumn{4}{|c|}{$\begin{array}{l}\text { Cantidad de personas que } \\
\text { hicieron la actividad o no }\end{array}$} & \multicolumn{3}{|c|}{$\begin{array}{c}\text { Motivos en actividades sin } \\
\text { hacer o hechas con dificultad }\end{array}$} \\
\hline $\mathrm{N}^{\circ}$ & $\begin{array}{l}\text { Le fue } \\
\text { simple }\end{array}$ & $\begin{array}{c}\text { Con } \\
\text { dificultad }\end{array}$ & $\begin{array}{c}\text { Sin } \\
\text { hacer }\end{array}$ & $\begin{array}{c}\mathrm{Se} \\
\text { desorientó }\end{array}$ & \begin{tabular}{|c|} 
No lo \\
encontró
\end{tabular} & $\begin{array}{c}\text { No lo } \\
\text { entendió }\end{array}$ \\
\hline \multirow{3}{*}{1} & \multicolumn{6}{|c|}{ Ingresar a GDrive y crear un documento } \\
\hline & $45 \%$ & $46,66 \%$ & $8,33 \%$ & $37,87 \%$ & $43,93 \%$ & $18,18 \%$ \\
\hline & 54 & 56 & 10 & 25 & 29 & 12 \\
\hline \multirow{3}{*}{2} & \multicolumn{6}{|c|}{$\begin{array}{l}\text { Escribir una nota para invitar a personas a un evento y } \\
\text { nombrarla "Invitación" }\end{array}$} \\
\hline & $21,66 \%$ & $19,16 \%$ & $59,16 \%$ & $22,34 \%$ & $69,14 \%$ & $8,51 \%$ \\
\hline & 26 & 23 & 71 & 21 & 65 & 8 \\
\hline \multirow{3}{*}{3} & \multicolumn{6}{|c|}{ Insertar una imagen relativa al evento } \\
\hline & $27,50 \%$ & $27,50 \%$ & $45 \%$ & $62,06 \%$ & $13,79 \%$ & $24,13 \%$ \\
\hline & 33 & 33 & 54 & 54 & 12 & 21 \\
\hline \multirow{3}{*}{4} & \multicolumn{6}{|c|}{$\begin{array}{l}\text { Compartir la invitación a una persona con permiso de } \\
\text { edición y a otra solo para comentar. }\end{array}$} \\
\hline & $10 \%$ & $25,83 \%$ & $64,16 \%$ & $43,51 \%$ & $24,07 \%$ & $32,40 \%$ \\
\hline & 12 & 31 & 77 & 47 & 26 & 35 \\
\hline \multirow{4}{*}{5} & \multicolumn{6}{|c|}{ Crear una carpeta llamada Trabajos en GDrive. } \\
\hline & $11,66 \%$ & $32,50 \%$ & $55,83 \%$ & $42,45 \%$ & $20,75 \%$ & $36,79 \%$ \\
\hline & 14 & 39 & 67 & 45 & 22 & 39 \\
\hline & \multicolumn{6}{|c|}{ Mover la invitación a la carpeta. } \\
\hline \multirow[t]{3}{*}{6} & $15,83 \%$ & $23,33 \%$ & $60,86 \%$ & $42,57 \%$ & $38,61 \%$ & $18,81 \%$ \\
\hline & 19 & 28 & 73 & 43 & 39 & 19 \\
\hline & \multicolumn{6}{|c|}{\begin{tabular}{|l} 
Descargar en el disco la invitación \\
\end{tabular}} \\
\hline \multirow[t]{3}{*}{7} & $34,16 \%$ & $39,16 \%$ & $26,66 \%$ & $37,97 \%$ & $22,78 \%$ & $39,24 \%$ \\
\hline & 41 & 47 & 32 & 30 & 18 & 31 \\
\hline & \multicolumn{6}{|c|}{$\begin{array}{l}\text { Buscar algún trabajo compartido por otro compañero y } \\
\text { averiguar a quienes más se les compartió dicho trabajo. }\end{array}$} \\
\hline \multirow[t]{3}{*}{8} & $12,50 \%$ & $30 \%$ & $57,50 \%$ & $37,14 \%$ & $29,52 \%$ & $33,33 \%$ \\
\hline & 15 & 36 & 69 & 39 & 31 & 35 \\
\hline & \multicolumn{6}{|c|}{ Dejar un comentario a algún trabajo que tenga permiso } \\
\hline \multirow[t]{2}{*}{9} & $15 \%$ & $56,66 \%$ & $36,66 \%$ & $42,85 \%$ & $16,07 \%$ & $32,14 \%$ \\
\hline & 18 & 68 & 44 & 48 & 18 & 36 \\
\hline \multirow{3}{*}{10} & \multicolumn{6}{|c|}{ Salir de la aplicación cerrando correctamente la sesión. } \\
\hline & $10,83 \%$ & $20 \%$ & $69,16 \%$ & $51,40 \%$ & $10,28 \%$ & $38,31 \%$ \\
\hline & 13 & 24 & 83 & 55 & 11 & 41 \\
\hline
\end{tabular}

Tabla 3: Resultados de eficacia y eficiencia Parte 1 y 2

La Tabla 4 muestra los resultados de la parte 3 de la planilla, en donde se registraron los tiempos de realización y grado de satisfacción de los participantes. 


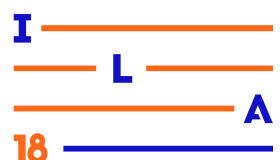

\begin{tabular}{|c|c|c|c|c|c|c|}
\hline & \multicolumn{3}{|c|}{ Tiempos de realización } & \multicolumn{3}{|c|}{ Grado de satisfacción } \\
\hline $\mathrm{N}^{\circ}$ & Rápido & Lento & Muy Lento & Alto & Medio & Bajo \\
\hline \multirow[t]{3}{*}{1} & \multicolumn{6}{|c|}{ Ingresar a GDrive y crear un documento } \\
\hline & $15,83 \%$ & $32,50 \%$ & $51,66 \%$ & $16,66 \%$ & $37,50 \%$ & $45,83 \%$ \\
\hline & 19 & & 62 & 20 & 45 & 55 \\
\hline 2 & \multicolumn{6}{|c|}{$\begin{array}{l}\text { Escribir una nota para invitar a personas a un evento y } \\
\text { nombrarla "Invitación" }\end{array}$} \\
\hline & $22,50 \%$ & $33,33 \%$ & $44,16 \%$ & $39,16 \%$ & $33,33 \%$ & $27,50 \%$ \\
\hline & 27 & 40 & 53 & 47 & 40 & 33 \\
\hline \multirow[t]{3}{*}{3} & \multicolumn{6}{|c|}{ Insertar una imagen relativa al evento } \\
\hline & $16,66 \%$ & $37,50 \%$ & $46,66 \%$ & $17,50 \%$ & $24,16 \%$ & $51,66 \%$ \\
\hline & 20 & 45 & 56 & 21 & 29 & 62 \\
\hline \multirow[t]{3}{*}{4} & \multicolumn{6}{|c|}{$\begin{array}{l}\text { Compartir la invitación a una persona con permiso de } \\
\text { edición y a otra solo para comentar. }\end{array}$} \\
\hline & $10,83 \%$ & $26,66 \%$ & $60,83 \%$ & $11,66 \%$ & $27,50 \%$ & $58,33 \%$ \\
\hline & 13 & 32 & 73 & 14 & 33 & 70 \\
\hline \multirow[t]{3}{*}{5} & \multicolumn{6}{|c|}{ Crear una carpeta llamada Trabajos en GDrive. } \\
\hline & $16,66 \%$ & $42,50 \%$ & $40,83 \%$ & $16,66 \%$ & $42,50 \%$ & $40,83 \%$ \\
\hline & 20 & 51 & 49 & 20 & 51 & 49 \\
\hline \multirow[t]{3}{*}{6} & \multicolumn{6}{|c|}{ Mover la invitación a la carpeta. } \\
\hline & $10 \%$ & $38,33 \%$ & $51,66 \%$ & $10 \%$ & $38,33 \%$ & $51,66 \%$ \\
\hline & 12 & 46 & 62 & 12 & 46 & 62 \\
\hline \multirow[t]{3}{*}{7} & \multicolumn{6}{|c|}{ Descargar en el disco la invitación } \\
\hline & $37,50 \%$ & $27,50 \%$ & $35 \%$ & $0,83 \%$ & $27,50 \%$ & $26,66 \%$ \\
\hline & 45 & 33 & 42 & 10 & 33 & 32 \\
\hline \multirow[t]{3}{*}{8} & \multicolumn{6}{|c|}{$\begin{array}{l}\text { Buscar algún trabajo compartido por otro compañero y } \\
\text { averiguar a quienes más se les compartió dicho trabajo. }\end{array}$} \\
\hline & $13,33 \%$ & $32,50 \%$ & $54,16 \%$ & $13,33 \%$ & $35 \%$ & $51,66 \%$ \\
\hline & 16 & 39 & 65 & 16 & 42 & 62 \\
\hline \multirow[t]{3}{*}{9} & \multicolumn{6}{|c|}{ Dejar un comentario a algún trabajo que tenga permiso. } \\
\hline & $27,50 \%$ & $35,83 \%$ & $36,66 \%$ & $24,16 \%$ & $50 \%$ & $25,83 \%$ \\
\hline & 33 & 43 & 44 & 29 & 60 & 31 \\
\hline \multirow[t]{3}{*}{10} & \multicolumn{6}{|c|}{ Salir de la aplicación cerrando correctamente la sesión. } \\
\hline & $28,33 \%$ & $36,66 \%$ & $35 \%$ & $25 \%$ & $43,33 \%$ & $31,66 \%$ \\
\hline & 34 & 44 & 42 & 30 & 52 & 38 \\
\hline
\end{tabular}

Tabla 4: Tabla de Resultados sobre Tiempos de Realización y Grado de Satisfacción

En la sección 3 se analiza con más detalle estos resultados cuantitativos y en la sección 4 se incluyen reflexiones sobre los comentarios vertidos en la parte 4.

\section{Análisis del testeo de usabilidad}

Las observaciones, los registros realizados durante el proceso de evaluación de la usabilidad y los resultados obtenidos, permiten realizar un análisis exhaustivo de la interacción entre el adulto mayor y las aplicaciones GDocs y GDrive y de las experiencias vivienciadas. De esto, se pueden destacar las siguientes apreciaciones.

Con respecto a la actividad 1, de ingresar a GDrive y crear un documento, un $46,66 \%$ de personas tuvieron dificultades que consistieron en no encontrar el programa. Tuvieron que recordar cómo se accede a las aplicaciones de Google, que al ser aplicaciones Web ya no debían recurrir al Menú de Inicio de la barra del sistema operativo.
A la mayoría les costó asociar las aplicaciones GDocs y GDrive con un prpdicin RL y los que ya estaban en la página de Google tatruaron en recordar que las aplicaciones se encontraban en el ícono \#. Asociar este ícono con un conjunto de aplicaciones, fue reconocido por muy pocos participantes. Sobre esto Etelvina de 75 años, expresó:_Esperen por favor. Cómo llegamos al programa ese? lo buscamos con el buscador Google pero no lo encontramos. No podemos arrancar aún.

A otras personas les confundió entender el sentido específico de cada una de las aplicaciones que utilizaron. Al principio dudaban si GDrive era para escribir o GDocs, ya que la creación la realizaron desde GDrive. Como lo indica Alberto de 81 años: _Entonces, para escribir voy a GDrive?

Estas complicaciones causaron demoras pero no decayó el grado de satisfacción porque estaban inicialmente muy motivados.

Con respecto a la actividad 2 de escribir una nota de invitación y colocarle un nombre al archivo, un $59,16 \%$ de personas no lo pudieron hacer, donde un $69,14 \%$ no encontraron esa opción. La mayoría no identificó que había que cliquear sobre el texto "Sin título" para colocarle uno. Algunos de los participantes intentaban a través de "Guardar como", pero esa opción no existe en las aplicaciones Web.

En la actividad 3 de insertar una imagen, la mayoría encontró la opción sin demora, pero le dio un error que no entendían, por tal motivo bajó el nivel de satisfacción de un $51,66 \%$ de personas. Ingresaron por la opción "Insertar imagen", luego por "Búsqueda" y en este caso, el sistema habilita imágenes que luego dan error de formato. La sucesión de pantallas se muestra en la Figura 1.

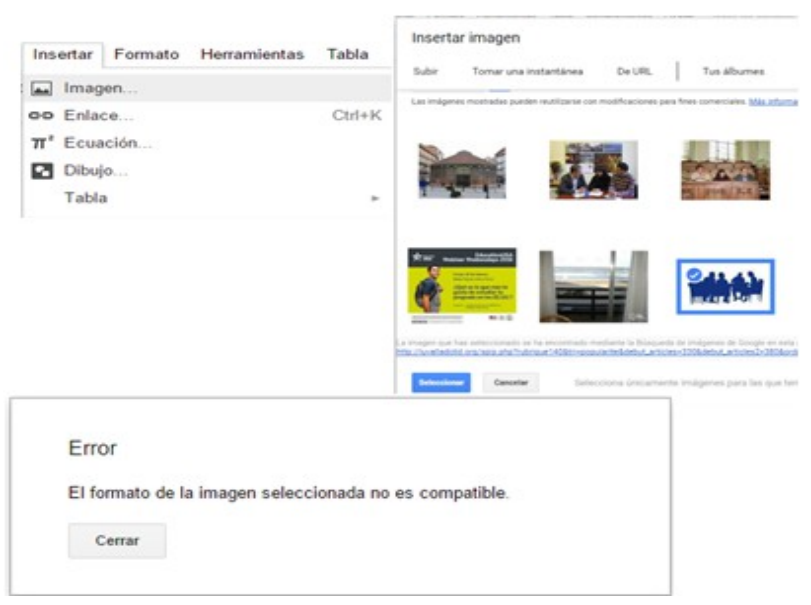

Figura 1: Error al Insertar Imagen 


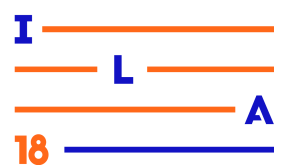

Al principio, los participantes manifestaron estar contentos por haberlo realizado en forma rápida, pero luego se confundieron al surgir este error, a pesar de haber observado un tilde de aprobación al lado de la imagen seleccionada. El comentario de Aldo de 81 años da cuenta de esta situación:

_Profesora, no sé qué hice mal, pero esto da error.

Esto demuestra que es inherente del usuario, especialmente de edad avanzada debido a su inseguridad en el manejo de la computadora, de responsabilizarse por todo, hasta de situaciones donde la persona no tuvo culpa ni responsabilidad. En la actividad 4 sobre compartir la nota realizada, un $64,16 \%$ de personas no lo pudo realizar. Hubo demoras y un $58,33 \%$ tuvo bajo nivel de satisfacción. Principalmente, se sintieron confundidos en lo que respecta a si compartían el documento o su enlace. No entendían la diferencia, ni tampoco se explica.

En la Figura 2, se muestran dos pantallas, una sin cliquear en "Obtener enlace para compartir" y la otra, donde se muestra cómo queda el cuadro de diálogo al cliquear en dicha opción.

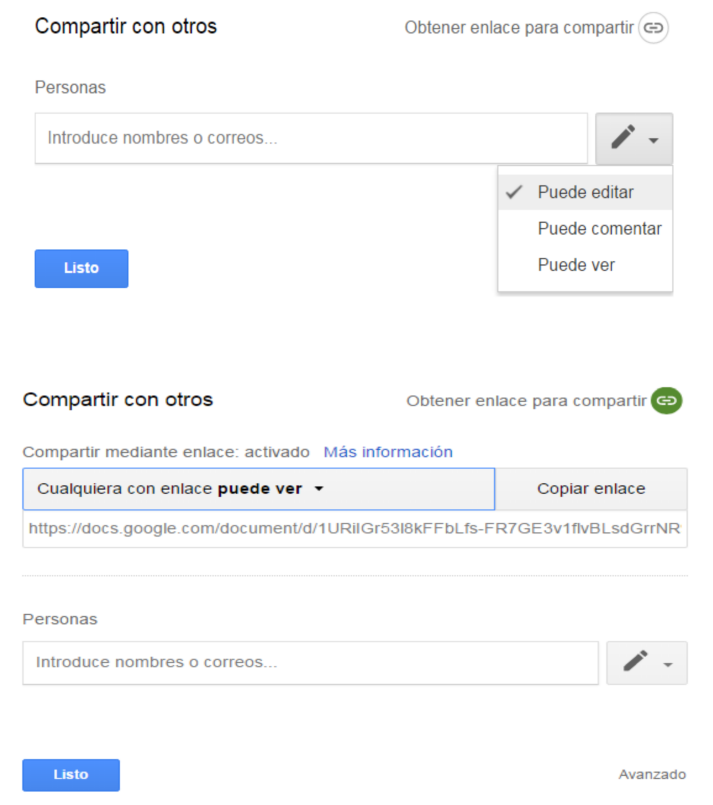

Figura 2: Ventanas de diálogo de Compartir sin y con la opción de Obtener enlace.

Al "compartir enlace" se presenta un menú con opciones de permisos de "ver", o "editar" al igual que el menú del casillero "Personas" que se encuentra debajo, y se confundieron sobre cuál de ellos utilizar para configurar los permisos de compartición. La expresión de Anselmo (78 años), expresa cómo el adulto mayor percibe esta situación:_Señorita, no entiendo. Hay dos formas de compartir?. Cuál debo utilizar?.

Para algunos se les hizo imposible concretar esta actividad debido a que esta ventana de diálogo presenta menús similares, y los participantes no sabían qué hacer, aumentando aún más su nivel de desorientación. Además, en "Obtener enlace para compartir" se confundieron aun más, ya que le costaba "salir" de ese contexto o cancelarlo. Lo único que tienen es la opción "Listo" con la cual, quedaba todo seteado para compartirlo sin poder revertirlo. Las cuestiones de diseño afectaron y entorpecieron la interacción de los usuarios. Todos dudaron y preguntaron las diferencias de estas opciones.

También, se complicaron al querer compartir a ciertas personas con permiso de "editar" mientras que a otras, con permiso de "comentar". El problema reside en el cuadro "Personas" como se muestra en la Figura 2, que indica "Introduce nombres o correos". Cuando uno introduce personas con sus nombres o correos, a todas ellas se le aplica un mismo tipo de permiso, denotados por el ícono de lápiz $\angle$. Se complica darles permisos distintos.

Para poder lograr cambiar de permiso a algunas y a otras no, el usuario tardó mucho tiempo, intentando varias veces, solicitando ayuda. Algunos eligieron "Listo" y volvieron a ingresar, otros introdujeron a todas las personas con un mismo permiso.

Muy pocas personas, unas 12, entraron por "Avanzado" para solucionarlo, donde se puede seleccionar cada persona en particular y determinar el tipo de permiso a otorgarle. Pero esto es un proceso muy complicado, implica razonamientos y estrategias que se obtienen recién al haber adquirido mucho dominio del tema o el poder animarse a probar. Uno de los comentarios registrados fue el de Carmen Antonia (68 años):_Dejo esta actividad Ivana, no sé cómo hacerla...sigo con el resto. No es que no entienda lo de compartir pero realmente no me sale, no puedo encontrarle la vuelta.

Otra de las dudas que le surgieron sobre esta actividad, y que la interfaz del usuario no brinda explicación sobre ello, es el nivel de propagación. Por ejemplo, lo que pregunta Hortensia (74 años) da cuenta de ello: el permiso de editar significa que puede quitar a personas a quien le compartí? o agregar otras?, o Nélida (77):_si le doy permiso 


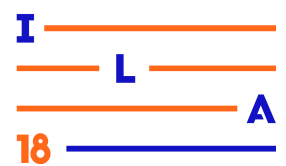

para editar y me lo borra? no solo el contenido, sino si me elimina el archivo entero? podría pasar eso. La falta de información también les molesta empeorando su nivel de satisfacción.

Las actividades 5 y 6 de "Crear carpeta" y "Mover el archivo" allí, tampoco fue fácil como ellos pensaban. Un $55,83 \%$ y un $60,86 \%$ de personas respectivamente abandonaron la tarea argumentando en su mayoría, desorientación. Los participantes lo intentaron realizar desde GDocs y otros, en GDrive.

Aquellos que pretendieron hacerlo desde GDocs, tuvieron algunos inconvenientes como el abrir un montón de pestañas. El problema reside dentro de la opción "Mover a...", que cerca de Mi Unidad se encuentra un ícono que ellos lo perciben como "crear carpeta" cuando en realidad es "abrir en pestaña nueva", como se muestra en la Figura 3.

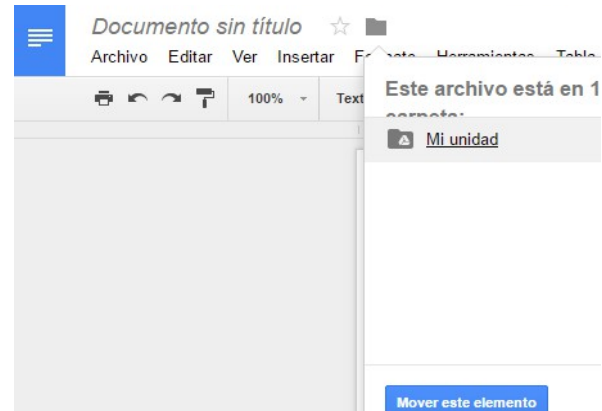

Figura 3: Icono que se confunde con Crear.

Esta situación condicionó la actividad 6, puesto que no quisieron volver a esta opción cuando era la mejor alternativa para "mover el archivo", finalidad que tenía dicha actividad.

Otros se inhibieron cuando se encontraron simultáneamente con dos mensajes contradictorios, por un lado el botón de "Mover" habilitado mientras que por el otro, se muestra un mensaje de "No se puede mover el archivo", como se muestra en la Figura 4. Nora, de 73 años, expresa: qué hago? aprieto igual en mover aunque salga ese mensaje?

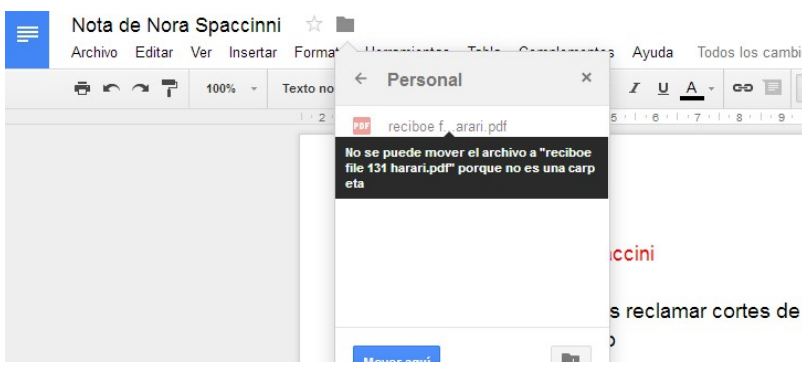

Figura 4: Mensaje de error al Mover aquí.
Siguiendo con la actividad de "Crear carpeta", otras personas intentaron hacerlo desde GDrive pero tuvieron inconveniente de regresar a dicha aplicación. Todos quisieron apretar en "volver", ya que desde GDrive activaron GDocs, pero habían perdido la sesión de la navegación porque GDocs inicialmente se había abierto en una pestaña nueva. $\mathrm{La}$ apertura de pestañas les confunde.

Otros apretaron el botón de rayas, $\equiv$ pero esto los llevó a la pantalla principal de GDocs nunca antes visitada, desorientándolos mucho ya que es un contexto que nunca habían ido antes. En la Figura 5, se muestra el archivo generado por la participante Olga que al apretar en dicho ícono, se encontró en una página desconocida.

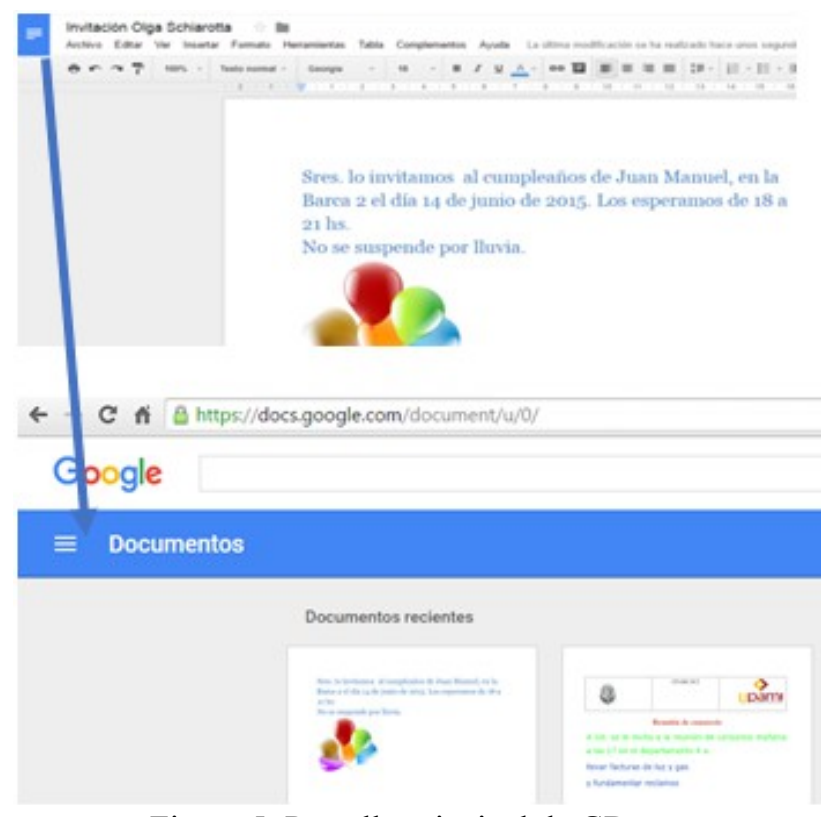

Figura 5: Pantalla principal de GDocs.

Muchos quisieron apretar de nuevo el ícono de rayas para regresar al documento, pero se abre un menú, y esto los desorientó aún más. Ver Figura 6.

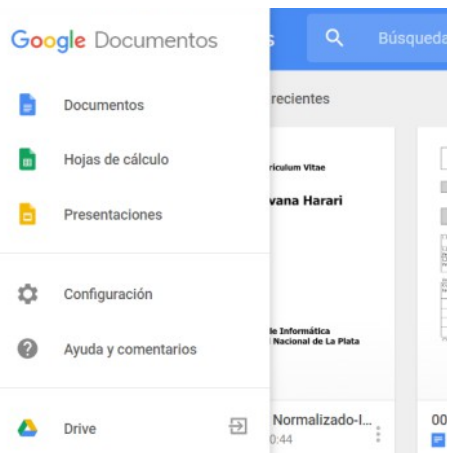

Figura 6: Menú de GDocs.

Aquellos que lograron volver a GDrive, les costó asociar "Nuevo" con "crear carpeta". Asociaban esta 


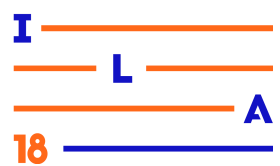

opción con crear documento en GDocs o en otras aplicaciones y no con manejo de disco.

En GDrive, también les costó el movimiento de archivo porque no encontraban el "Mover" que está dentro del icono de puntitos:

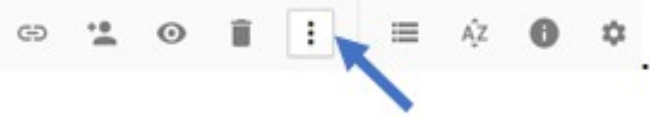

Los íconos no les resultaron naturales ni fáciles de reconocer ni recordar. Su diseño no permite un buen reconocimiento de las acciones que representan. Se quejaron de los tonos muy claros, del tamaño muy pequeño, y de su escasa información adicional. Con respecto a la actividad 7, la misma consistía en descargar la nota de invitación realizada anteriormente, en el disco o en un pendrive.

La mayoría, un 73,33\%, la pudo realizar pero les resultó muy diferente hacerlo desde GDrive que desde GDocs. Se quejaron de la dualidad y problemas de inconsistencias percibidos. Los confunde el poder hacer las mismas actividades desde dos programas muy conectados entre sí, pero que presentan distinto manejo y representación.

Aquellos que realizaron la descarga desde GDrive debieron seleccionar el archivo y utilizar una opción dentro del menú representado por "puntitos" como se visualiza en la Figura 7, que no es un diseño muy representativo. En este caso, el descargar, lo convierte automáticamente en docx, sin intervención del usuario.

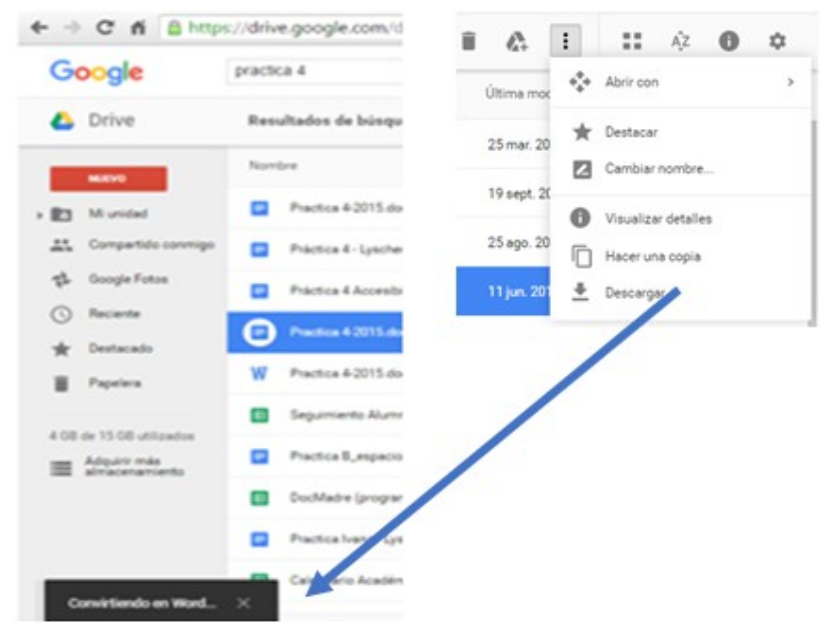

Figura 7: Descarga desde GDrive.

También, en la Figura 7 se observan claramente otros problemas de usabilidad. La opción de descarga se encuentra en la parte superior derecha, pero el mensaje sobre este proceso, en donde se indica que se está convirtiendo al formato docx, se muestra en forma truncada debajo y a la izquierda. Además en ningún momento se avisa progresividad, formas de cancelación, ni cuándo ni cómo finalizó dicho proceso.

Aquellos que realizaron la descarga desde GDocs, se dirigieron a la izquierda al menú archivo donde se encuentra la opción de Descargar como, habilitando la descarga en distintos formatos.

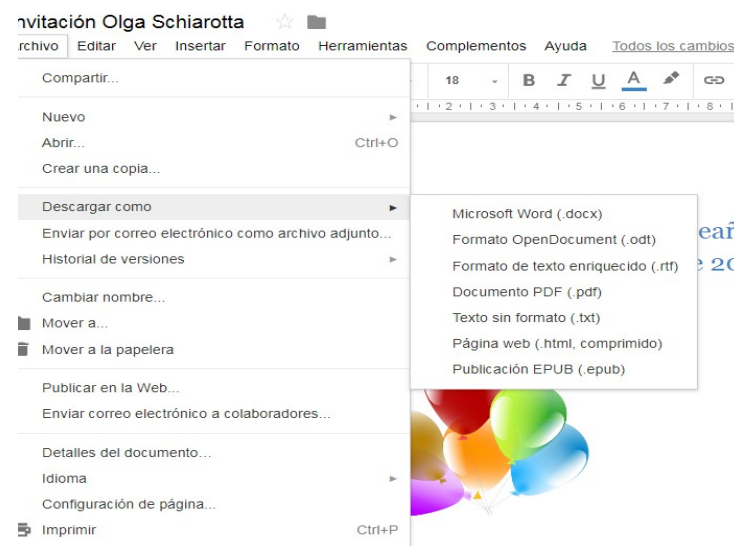

Figura 8: Descargar desde GDocs

Desde ambos programas, les costó comprender dónde quedó almacenado dicho archivo, pues no se les permitió elegir, ni tampoco se informó que quedó en la carpeta de Descargas del disco duro.

Con respecto a la actividad 8 sobre buscar trabajos compartidos por un determinado compañero y averiguar a quienes más se les compartió, un $57,50 \%$ lo abandonaron. Varios intentaron ir a GMail y acceder nuevamente al mail que habían recibido con el recurso compartido. Muchos no comprendieron que debían ir a la pestaña de GDrive y observar en la carpeta compartidos buscándolo allí. El tema es que no hay información relativa a esto, ni en GDrive ni en el mensaje que ellos recibieron por correo.

En GDocs, el tener que averiguar a quiénes se le compartió el trabajo, no recordaban si las caritas de arriba son los compartidos o los que están presentes simultáneamente, no hay información al respecto. El tener que ir y activar el botón "Compartir" para ver a quiénes se le compartió no les resultaba natural ni lógico, pensaban que esa opción servía para seguir compartiéndolo a otros más, pero no lo asociaban con la posibilidad de ver el autor y a quiénes se le compartió tal trabajo anteriormente.

Aquellos que intentaron realizarla desde GDrive, se ubicaron en archivos compartidos, y como el listado 


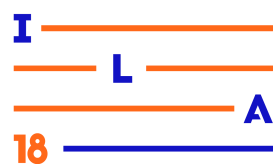

de archivos que tenían era corto, encontraron fácilmente los documentos compartidos. Tardaron en dilucidar a quienes más se les compartió. Para esto, debían ubicar el archivo, seleccionarlo, ir al ícono Información y observar que las caritas representan las personas a las que se le compartió, aunque esto no está explicitado, como se muestra en la Figura 9 .

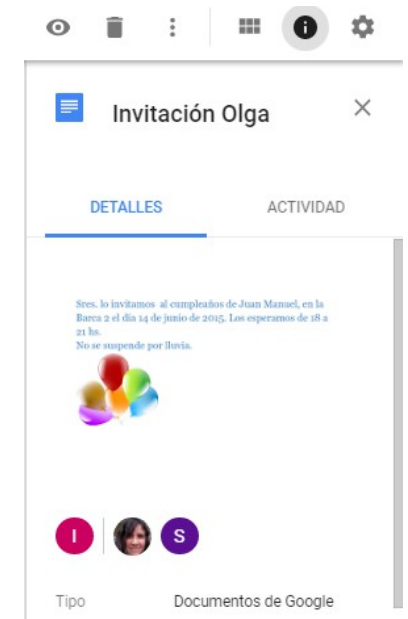

Figura 9: Icono Información

No hay explicación del sentido de las caritas, ni tampoco que se debe pasar el mouse por encima de los íconos para dilucidar el nombre de las personas. A pesar que están en la pestaña titulada Detalles, donde se encuentra más detalles es en la pestaña Actividad. Esto provoca insatisfacción como lo manifestaron un $51,66 \%$ de personas.

Hubo personas que ubicaron el archivo desde Acceso Directo, pero al cliquear en el mismo, no genera un cambio en el panel de información, como se muestra en la Figura 10.

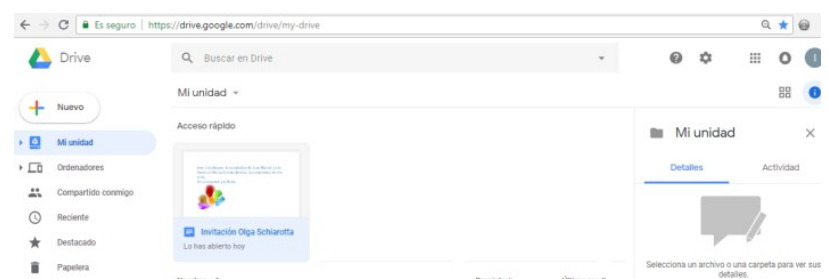

Figura 10: Documento seleccionado sin Información

Aquellas personas que fueron al mail, y abrieron el archivo desde allí, no se dieron cuenta que se abrió en el visor de Vista Previa. Esto significa que el archivo aún no fue abierto desde GDocs. En este contexto, el ver a quiénes se le compartió este trabajo también está en el "compartir", pero aquí se encuentra dentro del icono de puntitos verticales, como se muestra en la Figura 11.

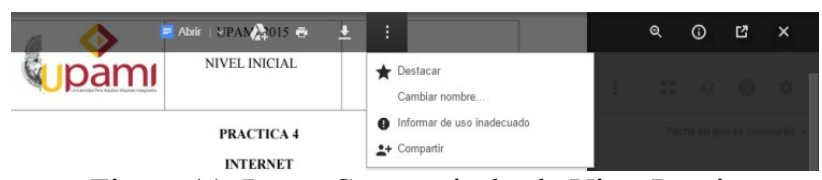

Figura 11: Icono Compartir desde Vista Previa

En definitiva, averiguar a quiénes fue compartido un trabajo fue una pregunta simple, conceptualmente entendible, pero llevarla a cabo no fue trivial. Hay alternativas pero todas implican procesos arduos de recordar. Esto fue realizado para los pocos afortunados, con el ícono ־ desde GDrive, otros con el ícono \& compantir desde GDocs y algunos con el ícono $\vdots$ desde el visor. Para una actividad que debe ser una de las más importantes, su diseño y manejo heterogéneos trae aparejado un sin fin de complicaciones innecesarias.

La actividad 9 consistía en dejar un comentario en un trabajo que se le había compartido al usuario. En el caso que el archivo compartido lo hayan descargado como un documento. docx, éste al querer accederse, se abre en el visor y hay que elegir abrir para lo abra GDocs y poder luego comentarlo.

Este proceso no está explicado en ningún lado. Supuestamente ellos lo ven abierto al documento. Algunos piensan que abrir es "abrir otro documento" como en Archivo-Abrir. Por ello, muchos se quedaron en este contexto y como unas 68 personas lo hicieron solicitando ayuda.

Muchos se confundieron y pensaron que el "Comentar" no se iba a encontrar dentro de la opción Comentarios, porque lo sentían como que eran comentarios ya hechos anteriormente o de otros. Y apretaron el ícono de mensajes por lo que ingresaron al chat erróneamente, como se aprecia en la Figura 12.

\section{Comentarios}

Figura 12: Botón Comentarios para comentar

Con respecto a la última actividad de salir de la aplicación, no advierten que hay más de una aplicación abierta, GDrive, GDocs para cada archivo abierto y GMail los que ingresaron para cumplir con una de las actividades. Muchos directamente salen de una de ellas pero dejan aplicaciones abiertas, otros cerraron el navegador 


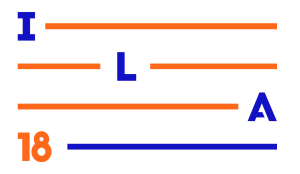

dejando todas las pestañas abiertas con sus respectivos programas con sesiones sin cerrar.

A algunas personas les apareció momentáneamente el siguiente mensaje y ninguno comprendió qué había sucedido, como se muestra en la siguiente figura.

\section{conoctanta a land}

Figura 13: Mensaje al cerrar sesión

\section{Reflexiones sobre los comentarios}

La parte 4 de la planilla de registro del testeo de usabilidad, estaba destinada para comentarios, sugerencias y opiniones de los participantes, frente a lo experimentado en el proceso de evaluación. Esto fue complementado con la última jornada realizada con ellos luego del testeo, que era el encuentro final de reflexión sobre la experiencia vivida. Esto dio lugar al debate, a la expresión de sus dichos y comentarios.

En este sentido y analizando los aspectos subjetivos de las personas a través de sus comentarios, se puede indicar las siguientes reflexiones.

Las expectativas iniciales sobre utilizar una aplicación popular y moderna fueron muy altas, por lo que tenían un gran entusiasmo inicial de trabajar con la computadora, y además como eran actividades conocidas como la de escribir, pensaban que iba a ser fácil. El entusiasmo y expectativas sobre experimentar una co autoría también fue muy motivador para ellos. Los dichos de Natalio (82 años) lo comprueba:_Es increible que con mi nieto estemos juntos escribiendo este trabajo cuando él está viviendo en el sur.

A pesar de esto, los problemas más graves de interacción estuvo más relacionado a problemas de ejecución que de comprensión.

Las quejas y comentarios estaban relacionados en su mayoría a que una vez encontrada la opción, les costaba llevarla a cabo. Hallaron complicaciones en su ejecución y no pudieron terminarla solos. Al no contar con información, ni sectores con títulos o buenos mensajes de aclaración o de error, se vieron la necesidad de cancelar la actividad o realizarla completamente con ayuda.
Se quejaron de encontrar una determinada actividad en un lugar como el mover pero después tardaron mucho en entender cómo había que manejar dicha acción. $\mathrm{O}$, al compartir a varias personas con distintos permisos, que lo localizaron fácilmente pero luego tardaron bastante en darse cuenta cómo había que proceder.

A pesar que a la mayoría les gustó el diseño simple de la interfaz, sugirieron letra más grande y colores no tan tenues, como lo expresó Marcos, de 69 años: _Vy a tener que cambiar los anteojos porque no los veo bien y no los distingo.

Otras de las causas de las quejas, fue los costos de intentar interpretar la iconografía utilizada que no reconocían bien los íconos o no son claros. A veces se trata del mismo ícono pero con distintas funciones como el ícono de puntitos, otras se encuentran en distinto lugar, como el ícono de compartir que está en la barra principal de GDrive pero dentro del ícono puntitos en el visor de documentos. Otras veces, lo notaban muy parecidos entre sí y no los podía distinguir, como lo indica Ismael, de 78 años, quien preguntó por qué hay dos botones con una i, o dos con puntitos?, donde el primer caso era el ícono de información y el otro era el de Ismael, o_esa barra es como el idioma chino para mí, son tōos signos parecidos, comentario de Yolanda con sus 88 años.

La sensación de insatisfacción se debe más que nada a entender lo que uno le solicita pero no poder encontrar las opciones, o en caso contrario, encontrarlas y no poder terminar de ejecutarlas por confusiones que les van surgiendo. Como lo indica Ilaria, de 74 años, cuando se le recordó que para ponerle el nombre al documento debía cliquear en Sin Nombre del documento: _Ah no! Jamás se me hubiera ocurrido que estuviera ahi!!

Otro de los problemas por lo que se quejaron, fue que el tratamiento y el diseño de funciones similares sean disímiles entre GDocs y GDrive. Los comentarios de Enriqueta de 77 años:_Por qué no ponen todo en un solo lugar y de la misma forma?!!, dan cuenta que esperan que las mismas funciones se encuentren en opciones representadas y manejadas de la misma forma, independientemente de la aplicación desde donde la lleven a cabo.

Cuando se sienten manipulados o controlados por la aplicación con secuencia de acciones sintácticas que no tienen lógica para ellos también es razón para 


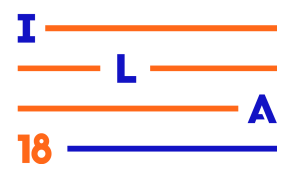

criticar el producto. Como por ejemplo para "ver a quienes se le compartió un documento " hay que ingresar a "compartir". Los comentarios de Rita y Alfonsina, de 68 y 75 años respectivamente lo demuestran:_Yo no quiero compartir nada, quiero ver a quien sé compartió nada más!!! y_Quién se iba a imaginar que debo compartir para ver los compartidos!!!.

Otro de los problemas que les disgusta es la falta de información o estar mal informados por la aplicación. Por ejemplo cuando tuvieron que abrir un trabajo de formato .docx cuando ya lo veían abierto. El tema es que se encuentran en un visor pero no está aún abierto en GDocs realmente. Esto no tiene información en ningún lado. Francesca (75 años) dijo:_No!! Abrir no! No quiero abrir otro documento.... O cuando tuvieron que ir a Avanzados del Compartir para darle distintos permisos a distintas personas a las que se le compartió. Esto no está informado o los mensajes presentes no son claros.

Otro de los puntos que manifestaron, fue que ellos esperan que la interfaz los guíe, les enseñe, que se considere que hay personas que aun no están preparados para explorar ni probar con prueba y error. Y peor aun cuando podes afectar un trabajo grupal o carpeta que está compartida.

Además, cuando intentan "explorar" les termina significando "empeorar". En el caso del ícono rayas que significó ir a la página principal de GDocs cuando ellos pensaban encontrar más opciones allí o volver a GDrive. Quisieron apretarlo de nuevo y se alejaron más pues lo llevó a un menú de opciones. El comentario de Nilda (72 años):_Noo!!! A dónde me metí??? Cómo vuelvo??.

Por último, se quejaron también cuando no entienden la terminología y las ayudas no explican sobre esto. Por ejemplo, lo que dijo Amanda con sus 83 años:_Resolver un comentario?? No será responder o leer dicho comentario?.

La ayudas son muy generales y no están diseñadas para asistir en un contexto de la interacción. No hay glosario terminológico ni tampoco explicación paso a paso de cómo resolver una actividad que involucra una transición de acciones en el producto.

Tampoco hay información explícita sobre la propagación, no se le detalla al usuario sobre qué se propaga frente al accionar individual sobre un recurso compartido. Como lo expresó claramente Luis, de 80 años:_Pude descargar el documento! pero se enteran que descargué el documento?, o Fanny de 72: _Si muevo esta invitación compartida los demás no lo van a encontrar!.

La falta de feedback y de transparencia, también son percibidos y cuestionados por el adulto mayor.

\section{Conclusiones}

Haber integrado una comunidad de adultos mayores en un proceso de evaluación de usabilidad de aplicaciones Google tan populares, fue una experiencia trascendental y muy enriquecedora. El hecho que los participantes supieran que el testeo era para analizar las aplicaciones y no a ellos específicamente, fue altamente positivo. Ellos adquirieron confianza y soltura para expresar sus miedos, desconfianzas, inseguridades, dudas.

El sentido del humor, las ganas de aprender también fue muy gratificante. El participar en un proceso de evaluación donde se analizaba las falencias del producto más que en la de ellos mismos, le resultó algo novedoso y entretenido._No podemos creer que hemos aprendido y encima colaborado en un proceso de testing!, mencionó Gladys con 78 años de edad. O las palabras de Noelia de 71 años _Aprendimos que no somos los responsables de todos los errores que cometemos en el software. Increíble! los desarrolladores del producto también tienen la culpa!.

Al trabajar con adultos mayores se potencia enormemente la parte emocional y social, sus sentimientos y deseos de participar en estas relaciones sociales virtuales, juegan un papel fundamental en la interacción y por consiguiente en el desarrollo del test.

Presentaron muchas capacidades relevantes a la hora de evaluar productos de software, como su sentido común, su capacidad para detectar inconsistencias en el lenguaje, en el diseño, identificando claramente situaciones donde no se detectaba una lógica determinada o una deliberación previa.

Es importante considerar el concepto del tiempo que tienen, ellos se toman tiempo con gusto para leer la pantalla, para leer los mensajes, por lo que perciben fácilmente falta de información o información inútil, incoherencias lingüísticas o expresiones erróneas. Las cuestiones de eficiencia como los tiempos de 


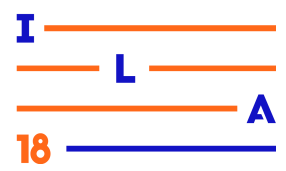

realización no los afecta en su grado de satisfacción. La satisfacción la relacionan más con entender qué hacer y que la interfaz lo ayude y acompañe en esa intención.

Debido a la edad y a los tiempos con que ellos se manejan, hubiera sido demasiado rápido y riguroso realizar el test de usabilidad en una forma tradicional y aislada, en unas pocas horas. La planificación de varios encuentros, con charlas introductorias sobre el producto y sobre el testeo, las entrevistas y cuestionarios, dos o tres sesiones para el desarrollo del testeo, y el encuentro final fue fundamental para los logros obtenidos y el éxito de la experiencia, tanto por el nivel de participación de los usuarios como de la fuente de información y de contribuciones adquiridas a través de ellos.

Acá se percibió la constitución de una comunidad entre los adultos mayores y expertos en HCI, donde la retribución fue mutua. En este proceso se invitó al diálogo y a la participación, se incentivó el debate e inclusive se incitó a la generación de ideas y sugerencias de cambio del diseño.

Fue una experiencia muy satisfactoria desde lo pedagógico, social como desde los aportes significativos en el área de HCI.

\section{Referencias Bibliográficas}

CASAMAYOU, Adriana y MORALES G. M. Julia. Personas mayores $y$ tecnologías digitales: desafíos de un binomio. Psicología, Conocimiento y Sociedad 7(2), 199-226, Uruguay. (2017).

DIAZ, F.Javier; BANCHOFF, C.; HARARI, V.; HARARI, I. y AMBROSI, V. Articulating two social based informatics projects. Invoice for ICIT, International Conference on Informatics Technologies, en Saratov, Rusia. (2012).
GREMILLION, B.; CHAPMAN, C. y MUNROE, L. A Field Guide to Usability Testing. Samshing Magazine. (2012).

HASSAN Montero Y., y ORTEGA Santamaría S. Informe APEI de usabilidad. Gijón: Asociación Profesional de Especialistas en Información. (2009).

ISO 9241-11:2018. Ergonomics of human-system interaction Part 11: Usability: Definitions and concepts. International Organization for Standarization. (2018).

KRUG, Steve. No me hagas pensar. Don't make me think!: A common sense approach to Web usability. Prentice Hall. (2006).

MANSOR, A.Z. Google GDocs as a Collaborating Tool for Academicians. Procedia-Social and Behavioral Sciences Vol.59. (2012).

NIA y NLM. Making your Web site seniorfriendly: A checklist. National Institute on Aging and National Library of Medicine. (2002).

NIELSEN, J. y MACK, R.. Usability Inspection Methods. John Valley and Sons Ed. (2004).

FINN, Kate y JHONSON, Jeff. Designing for Older Adults: Usability Considerations for Real Users. Disponible en www.wiserusability.com /author/adminjeff/.

\section{Agradecimientos}

Se agradece profundamente a las 120 personas que participaron en el testeo de usabilidad. Por su amabilidad, buena predisposición y voluntad permanentes. 